\title{
Regulation of mammalian pyruvate dehydrogenase complex by phosphorylation: complexity of multiple phosphorylation sites and kinases
}

\author{
Mulchand S. Patel ${ }^{1,2}$ and Lioubov G. Korotchkina ${ }^{1}$ \\ ${ }^{1}$ Department of Biochemistry, School of Medicine and Biomedical \\ Sciences, State University of New York at Buffalo, Buffalo, New \\ York 14214, USA \\ ${ }^{2}$ Corresponding author: Tel, +1-716-829-3074; \\ Fax, +1-716-829-2725; E-mail, mspatel @buffalo.edu
}

Accepted 21 November 2001

Abbreviations: PDC, pyruvate dehydrogenase complex; E1, pyruvate dehydrogenase; E2, dihydrolipoamide acetyltransferase; E3, dihydrolipoamide dehydrogenase; E3BP, E3-binding protein; PDK, pyruvate dehydrogenase kinase; PDP, phospho-pyruvate dehydrogenase phosphatase; TPP, thiamine pyrophosphate; HE=TPP, 2- $\alpha$ hydroxyethylidene-TPP; BCKDH, branched-chain $\alpha$-keto acid dehydrogenase; DCPIP, 2,6-dichlorophenolindophenol.

\begin{abstract}
This review summarizes the recent developments on the regulation of human pyruvate dehydrogenase complex (PDC) by site-specific phosphorylation by four kinases. Mutagenic analysis of the three phosphorylation sites of human pyruvate dehydrogenase (E1) showed the site-independent mechanism of phosphorylation as well as site-independent dephosphorylation of the three phosphorylation sites and the importance of each phosphorylation site for the inactivation of E1. Both the negative charge and size of the group introduced at site 1 were involved in human E1 inactivation. Mechanism of inactivation of E1 was suggested to be site-specific. Phosphorylation of site 1 affected E1 interaction with the lipoyl domain of dihydrolipoamide acetyltransferase, whereas phosphorylation site 3 appeared to be closer to the thiamine pyrophosphate (TPP)-binding region affecting coenzyme interaction with human E1. Four isoenzymes of pyruvate dehydrogenase kinase (PDK) showed different specificity for the three phosphorylation sites of E1. All four PDKs phosphorylated sites 1 and 2 in PDC with different rates, and only PDK1 phosphorylated site 3. PDK2 was maximally stimulated by the reduction/acetylation of the lipoyl groups of E2. Presence of the multiple phosphorylation sites and isoenzymes of PDK is important for the tissue-specific regulation of PDC under different
\end{abstract}

physiological conditions.

Keywords: pyruvate dehydrogenase, thiamine pyrophosphate, phosphorylation, isoenzymes of kinase

\section{Introduction}

Pyruvate dehydrogenase complex (PDC) plays a key role in glucose metabolism by catalyzing oxidative decarboxylation of pyruvic acid and providing acetyl-CoA and NADH for the tricarboxylic acid cycle and biosynthetic processes (Figure 1). Eukaryotic PDC is a highly organized multienzyme complex with the core structure formed by 60 subunits of dihydrolipoamide acetyltransferase (E2) and 12 monomers of dihydrolipoamide dehydrogenase-binding protein (E3BP) to which other components of the complex are bound: 20-30 heterotetramers $\left(\alpha_{2} \beta_{2}\right)$ of pyruvate dehydrogenase (E1), 6-12 homodimers of dihydrolipoamide dehydrogenase (E3), 1-2 homo (or hetero) dimers of pyruvate dehydrogenase kinase (PDK) and 2-3 heterodimers of phosphopyruvate dehydrogenase phosphatase (PDP) (Figure 2) (Patel and Roche, 1990). Detailed information of the structure and regulation of PDC may be found in several recent reviews (Sugden and Holness, 1994; Patel and Harris, 1995; Perham, 2000; Harris et al., 2001; Reed, 2001; Roche et al., 2001).

E1 catalyzes the first irreversible and rate-limiting step in the PDC catalyzed reactions, i.e. the thiamine pyrophosphate (TPP)-dependent decarboxylation of pyruvic acid with formation of 2- $\alpha$-hydroxyethylidene-TPP (HE= TPP) and carbon dioxide and reductive acetylation of lipoyl moieties of E2. Heterotetrameric E1 has two active sites that interact with each other during catalysis (Butler et al., 1977; Khailova et al., 1982). The threedimensional structures of branched-chain $\alpha$-keto acid dehydrogenase (BCKDH) from Pseudomonas putida and human having high homology with human E1 of PDC ( $41 \%$ for $\alpha$ subunit and $52 \%$ for $\beta$ subunit for human $\mathrm{BCKDH}$ ) showed that each of four subunits of $\mathrm{BCKDH}$ interacts with each other by extensive surface contacts (Aevarsson et al., 1999; Aevarsson et al., 2000). The active sites of BCKDH are composed of amino acid residues of both the $\alpha$ and $\beta$ subunits providing " $\mathrm{V}$ shaped" conformation of TPP necessary for catalysis. $\mathrm{BCKDH}$ binds to the binding domain of branched-chain dihydrolipoamide acyltransferase through the C-terminal 


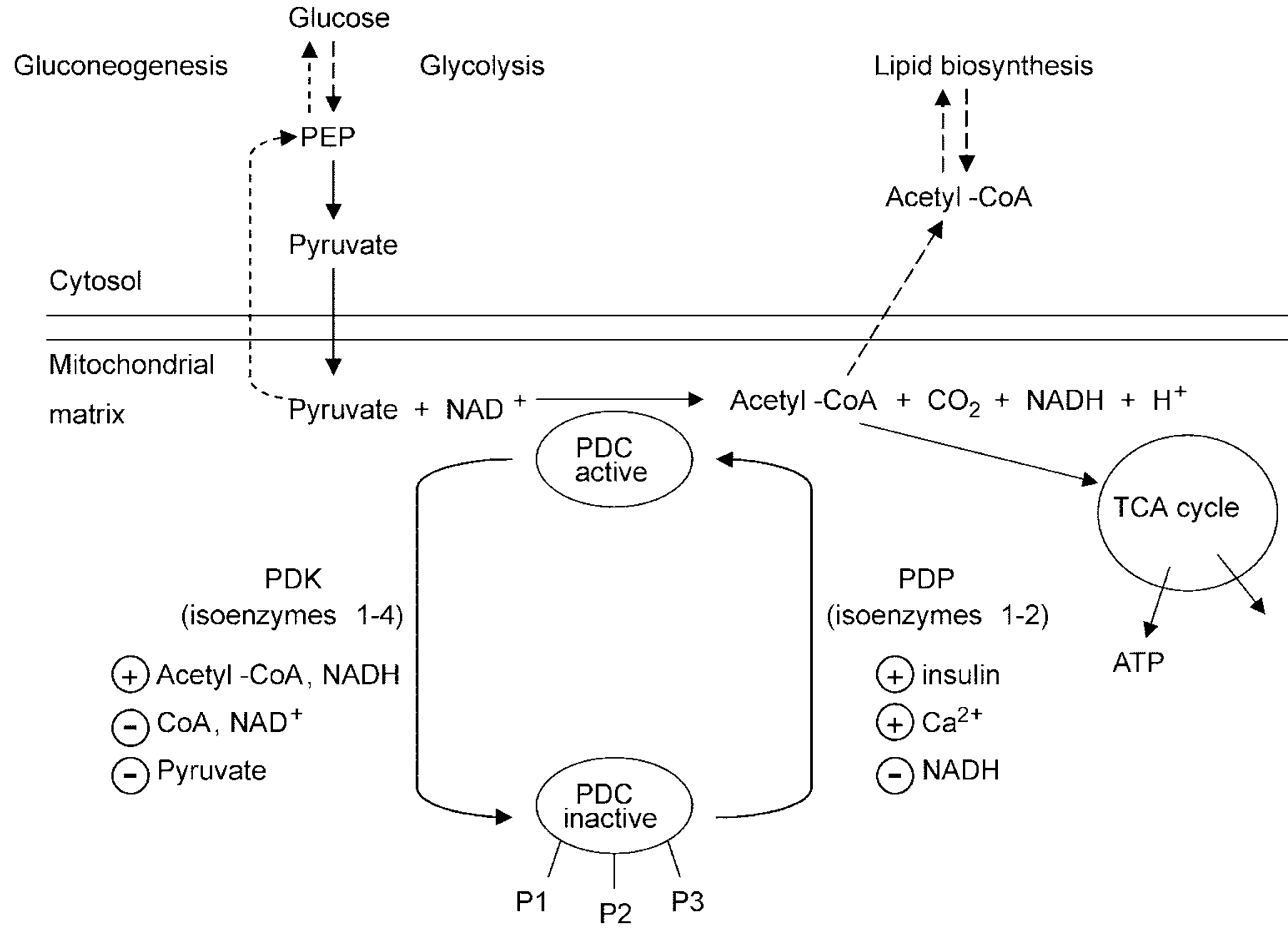

Figure 1. Regulation of PDC activity by interconversion between active (unphosphorylated) and inactive (phosphorylated) forms catalyzed by PDPs and PDKs. Three phosphorylated sites of E1-PDC are shown as P1, P2 and P3.

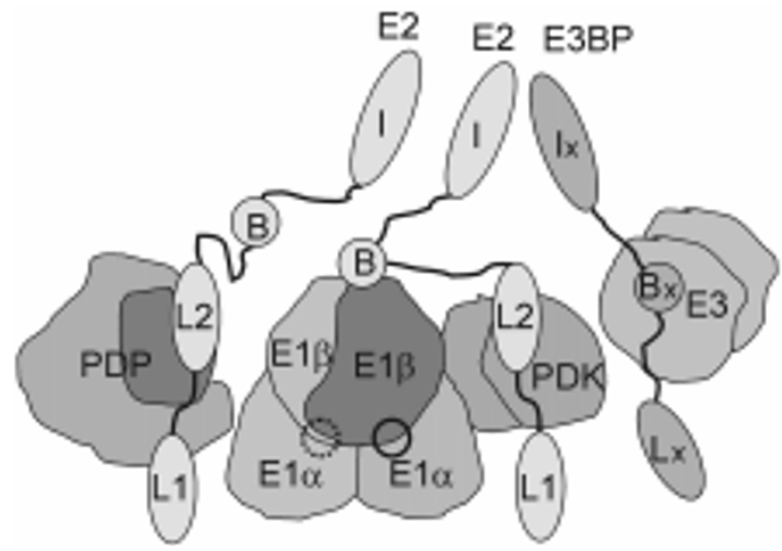

Figure 2. Schematic presentation of the domains of the PDC components and their sites of interaction. Only two molecules of E2 and one molecule of each of E1, E3, E3BP, PDK and PDP are shown. E2 consists of several domains: I, inner domain; B, binding domain; L2 and L1, lipoyl domains. E3BP consists of: Ix, inner domain; Bx, binding domain, Lx, lipoyl domain. E1 $\left(\alpha_{2} \beta_{2}\right.$ tetramer) is bound to the binding domain of E2, and E3 to the binding domain of E3BP. Both PDK and PDP are bound through the lipoyl domains of E2. One black circle and dotted circle identify position of two E1 active sites [based on the three-dimensional structures of human and Pseudomonas putida BCKDH (Aevarsson et al., 1999; Aevarsson et al., 2000)].

part of its $\beta$ subunits (Aevarsson et al., 1999). A similar assembly most probably occurs for PDC-E1.

\section{Regulation of PDC activity}

Activity of higher eukaryotic PDC is regulated in a large part by interconversion of the $\mathrm{E} 1$ component between an active (nonphosphorylated) form and inactive (phosphorylated) form. This interconversion is catalyzed by PDC-specific PDK and PDP. (Figure 1). Three serine residues were identified as phosphorylation sites in the $E 1 \alpha$ subunit of bovine and porcine E1s and designated site 1 (Ser264 in human E1 $\alpha$ sequence), site 2 (Ser271) and site 3 (Ser203) (Yeaman et al., 1978; Dahl et al., 1987). These three sites are present in both human and mouse E1 isoforms (somatic and testis-specific), somatic isoform of rat E1, isoform II of Ascaris suum E1, and Drosophila melanogaster E1; however, only two sites (site 1 and site 2) are present in rat E1 (testis-specific isoform), isoform I of Ascaris suum E1, in Caenorhabditis elegans. Only site 1 is found in the sequence of plants (Arabidopsis thaliana, Zea mays, Pisum sativum, Solanum tuberosum) and yeast E1 (GenBank). Phosphorylation of site 1 correlates with major inactivation of the complex. Half-of-the-site reactivity is exhibited by E1 as phosphorylation of only one of six serine residues in the two $\alpha$ subunits is sufficient for complete inactivation of the complex (Sugden and Randle, 1978).

Regulation of glucose metabolism in the cells depends in part on activity of PDC. Several isoenzymes of PDK 
are present in humans, rodents, plants but only one in nematodes and fruit fly (Gudi et al., 1995; Rowles et al., 1996; Katsube et al., 1997; Chen et al., 1998; Thelen et al., 1998; Thelen et al., 2000). PDKs show very little amino acid sequence similarity with eukaryotic Ser/Thrprotein kinases and were suggested to belong to the ATPase/kinase superfamily (Bowker-Kinley and Popov, 1999). Both PDKs and PDPs are bound to the lipoyl domains of E2 in PDC (Yang et al., 1998). The shortterm regulation of PDC activity is achieved through modulation of PDK activity (and correspondingly the level of PDC phosphorylation) through the changes in the status of the lipoyl groups from oxidized to reduced and acetylated forms (Ravindran et al., 1996). The longterm regulation of PDC is exerted at the transcriptional level by increased expression of PDK (leading to hyperphosphorylation of PDC) in response to different pathological conditions (diabetes, starvation). Mammalian PDK isoenzymes are expressed in a tissue-specific manner with PDK1 present mostly in the heart, PDK2 in most of the tissues, PDK3 in testis and PDK4 having the higher amounts in heart and skeletal muscle (Bowker-Kinley et al., 1998). The tissue-specific expression of PDK isoenzymes, their different specific activity and different sensitivity towards effectors and metabolites provide a coordinated regulation of glucose metabolism and hence glucose homeostasis in the organism.

\section{Specificity of the three phosphorylation sites of human E1}

Studies performed on the PDC from mammalian tissues have revealed that the rate of phosphorylation is different for the three sites with site 1 phosphorylated faster than sites 2 and 3 (Yeaman et al., 1978). Also the extent of phosphorylation of the three sites in vivo was different with the maximum incorporation of phosphoryl groups into site 1 (Sale and Randle, 1982). To distinguish among the three phosphorylation sites, several mutants of recombinant human E1 (replacing serines of the phosphorylation sites with alanine) were generated with a single mutation at site $1(1 \mathrm{~A})$, site $2(2 \mathrm{~A})$ or site $3(3 \mathrm{~A})$; double mutations leaving only one site available for modification, site 1 (2A3A), site 2 (1A3A) or site 3 (1A2A). All mutant E1s were phosphorylated by the E2-E3BPPDK subcomplex purified from bovine heart (Korotchkina and Patel, 1995). Phosphorylation resulted in concomitant inactivation of E1 as measured by E1 activity in the PDC reaction after reconstitution with E2-E3BP and E3. The findings of this study indicated that (i) phosphorylation was not sequentially ordered and three sites could be modified either individually or in the presence of the other sites, (ii) modification of each of the three phosphorylation sites caused E1 inactivation, and (iii) the rates of phosphorylation and inactivation of human
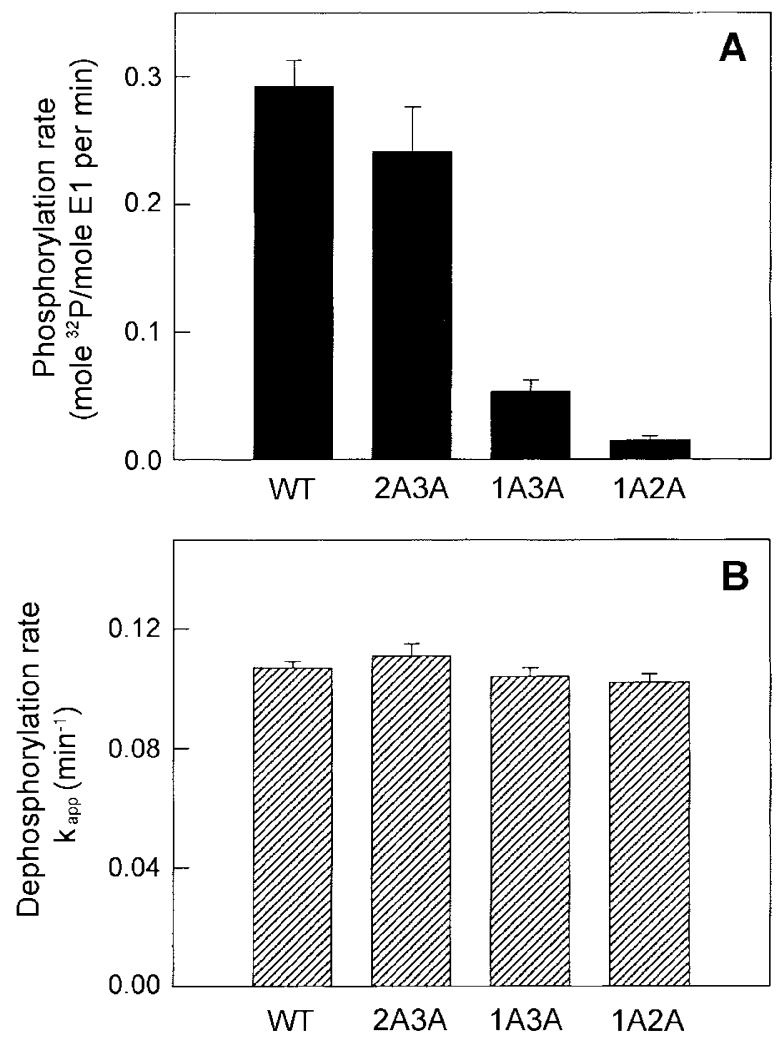

Figure 3. Rates of phosphorylation $(A)$ and dephosphorylation $(B)$ of the wild-type and mutant human E1s. A: The rates of phosphorylation were determined from the initial slopes of ${ }^{32} \mathrm{P}$ incorporation during phosphorylation of the wild-type and mutant human E1s by the bovine E2-E3BP-PDK subcomplex in the presence of $40 \mu \mathrm{M}\left[\gamma^{32}\right.$ P]ATP (Korotchkina and Patel, 1995). B: $k_{\text {app }}$ of dephosphorylation were calculated from semilog plots of the time courses of ${ }^{32} \mathrm{P}$ release during dephosphorylation of the wild-type and mutant E1s by the PDP (Korotchkina and Patel, 1995).

recombinant E1s were site-specific. Figure $3 \mathrm{~A}$ compares the rates of phosphorylation of the three sites when present individually, demonstrating that the rate of phosphorylation of site 1 was 4.5 -fold higher than of site 2 and 16 -fold higher than of site 3 . The rates of dephosphorylation, on the contrary, appeared to be nearly identical for sites 1,2 and 3 present either individually or in combinations with the other sites (Figure 3B), indicating a random mechanism of dephosphorylation of the three sites (Korotchkina and Patel, 1995).

Besides mammalian E1, study on the role of multiple phosphorylation sites in PDC was performed only on Ascaris suum E1-I isoform, having two phosphorylation sites (Huang et al., 1998). Similar to human E1, site 1 of nematode $\mathrm{E} 1$ had higher rate of modification than site 2 and phosphorylation of each of the two sites caused inactivation of E1. In contrast to PDC-E1, site 2 of $\mathrm{BCKDH}$ did not take part in the regulation of $\mathrm{BCKDH}$ activity (Zhao et al., 1994). Position of site 1 (Ser292) of $\mathrm{BCKDH}$ in the sequence is conserved with the Ser264 (site 1) of PDC-E1, while position of site 2 is not. 


\section{Mechanism of inactivation of human E1 by phosphorylation of three sites}

The studies on the mechanism of inactivation of mammalian and avian E1s showed the effect of E1 phosphorylation on the affinity for TPP and the formation of enzyme-bound HE=TPP from E1-TPP in either the forward (pyruvate as a substrate) or reverse reaction (acetylhydrolipoate as a substrate) (Roche and Reed, 1972; Walsh et al., 1976; Butler et al., 1977; Korotchkina et al., 1995; Nemerya et al., 1996). In a recent study the mechanism of inactivation of human E1 was investigated using site-specific replacement of the three phosphorylation sites (Korotchkina and Patel, 2001a). Serines of the phosphorylation sites 1 (Ser264), 2 (Ser271) and 3 (Ser203) were replaced with alanine, glutamate and glutamine. Figure 4 demonstrates the activities of
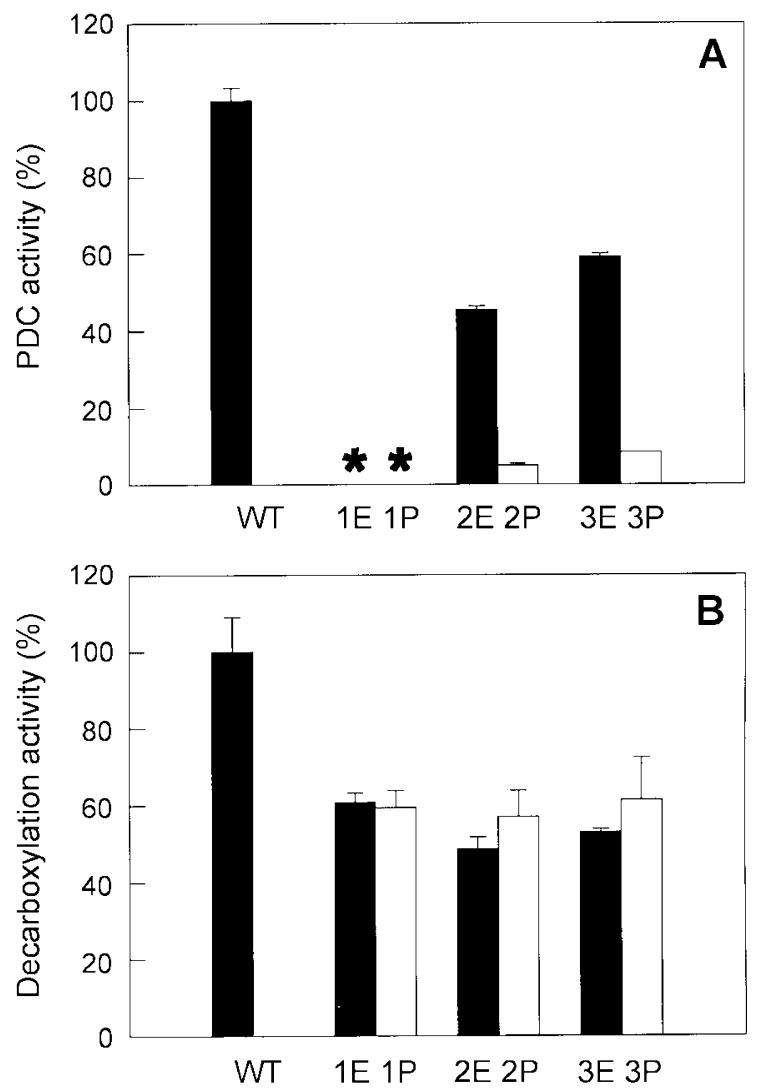

Figure 4. Activities of human E1s with one of the three phosphorylation sites substituted with glutamate or phosphorylated. A: Activities were measured by the PDC assay (complete PDC reaction) by NADH formation. B: Activities were measured by the $\mathrm{CO}_{2}$ assay (decarboxylation reaction) by the formation of ${ }^{14} \mathrm{CO}_{2}$ from $\left[1-{ }^{14} \mathrm{C}\right]$ pyruvate in the absence of electron acceptors. 2A3A E1 was phosphorylated on site 1 only (1P) by PDK2, 1A3A $\mathrm{E} 1$ on site 2 only (2P) by PDK2 and 1A2A E1 on site 3 only (3P) by PDK1 in the presence of human E2-E3BP (Korotchkina and Patel, 2001a). Black bars, wild-type and glutamate-substituted mutant $\mathrm{E} 1 \mathrm{~s}(1 \mathrm{E}, 2 \mathrm{E}$ and $3 \mathrm{E})$, and open bars, double mutants of E1 phosphorylated on the remaining phosphorylation site (1P, 2P and 3P)(Korotchkina and Patel, 2001a). *, not detectable. the glutamate-substituted mutants of sites 1,2 and 3 in comparison with the E1s phosphorylated on sites 1, 2 and 3 in PDC-assay (reconstituted PDC-reaction) and $\mathrm{CO}_{2}$-assay (decarboxylation reaction only). The glutamate mutant of site 1 (1E) displayed the same behavior as the $\mathrm{E} 1$ phosphorylated only on site 1, i.e. the absence of PDC activity (Figure 4A) and moderate reduction in the decarboxylation reaction (Figure 4B). These results indicated that probably the second partial E1 reaction (i.e. reductive acetylation of lipoyl groups of E2) is affected to a greater extent by phosphorylation than the decarboxylation step. 1Q E1 had only $3 \%$ of control activity in PDC-reaction (Korotchkina and Patel, 2001a) indicating the importance of the volume of the substituted group as well as the charge. Substitutions of sites 2 and 3 with glutamate did not result in the same degree of inactivation as the phosphorylation of sites 2 and 3 did (Figure 4B). Some compensation for the loss of activity could be explained by the differences in the local conformation of the site 2 and site 3 after folding of the mutated E1s.

Substitution mutants of site 1 (1A, 1E, 1Q) had higher $\mathrm{K}_{\mathrm{m} \text {,app }}$ for pyruvate (2.6 to 4.5 -fold) than the wild-type $\mathrm{E} 1$, as determined in the 2,6-dichlorophenolindophenol (DCPIP)assay (because of the lack or negligible activity of the mutants of site 1 in the PDC assay). However, a similar substitution in the nematode PDC-E1 resulted in a decrease in activity without a change in $\mathrm{K}_{\mathrm{m} \text {,app }}$ for pyruvate (Huang et al., 1998). Mutants of sites 2 and 3 did not display any significant difference in the $\mathrm{K}_{\mathrm{m} \text {,app }}$ for pyruvate. Site 1 is localized in the substrate channel according to the structure of BCKDH and may take part in positioning of the substrates during the catalytic reaction (Aevarsson et al., 1999). For this reason the replacement of serine even with alanine resulted in the modified affinity for the first substrate, pyruvate (Korotchkina and Patel, 2001a).

It is interesting to note that when Ser264 (site 1) is replaced with glutamine the activity with pyruvate is decreased $\sim 40 \%$, whereas the activity with a substrate analog, $\alpha$-ketobutyrate, was increased by about 5 -fold. The modest increase in activity in the DCPIP assay with $\alpha$-ketobutyrate was seen with $1 \mathrm{~A}(168 \%)$ and $1 \mathrm{E}$ (190 $\%)$ mutant E1s. These findings indicated that serine could be involved in formation of the active site and its elimination (even replacement with alanine having similar size) could change the substrate channel. The presence of the larger side chain (glutamine) could possibly affect the structure of the active site even more allowing a bulkier substrate analog to be processed. However, the second partial reaction (reductive acetylation) could not be catalyzed efficiently (1Q had only about 3\% activity in the PDC reaction). Phosphorylation of E1 may affect the interaction with the second substrate more than that with the first (pyruvate and its analog). The acidic residues of the lipoyl domain were found to be important 
for interaction with E1 (Baker et al., 2000). The phosphate group in the $E 1$ active site could affect $E 1$ interaction with the negatively charged residues of the lipoyl domain because of its size and anionic nature (Liu et al., 2001). The impact of E1 phosphorylation on the second E1-catalyzed reaction of reductive acetylation was recently reported by Liu et al. (Liu et al., 2001) confirming these observations.

Kinetic analysis revealed that $\mathrm{K}_{\mathrm{m} \text {,app }}$ values for TPP were increased for $2 \mathrm{E}$ (10-fold) and $3 \mathrm{E}$ (27-fold). $\mathrm{K}_{\mathrm{i}}$ for pyrophosphate (competitive inhibitor of $E 1$ with respect to TPP) increased for $2 \mathrm{E}$ and $3 \mathrm{E}$ 7-8-fold compared to the wild-type $\mathrm{E} 1$. These results indicated that phosphorylation of site 3 and to a lesser extent site 2 may affect TPP binding. On the other hand TPP protected the phosphorylation sites from modification (Korotchkina and Patel, 2001a). The $k_{a p p}$ of inactivation decreased in the presence of TPP by about 4-fold for site 1, 7-fold for site 2 and 40 -fold for site 3 . Site 3 is localized most probably closer to binding of the pyrophosphate moiety of TPP and hence the incorporation of the negatively charged bulky phosphoryl-group could interfere with TPP binding.

\section{Site-specificity of four PDK isoenzymes}

Recently we (Korotchkina and Patel, 2001b) and others (Kolobova et al., 2001) have addressed the question of the site-specificity of PDK isoenzymes towards the three phosphorylation sites of E1. In our studies activities of four PDK isoenzymes were determined using double site mutant E1s having only one phosphorylation site available: $2 A 3 A$ with site 1 only, $1 A 3 A$ with site 2 only, and $1 \mathrm{~A} 2 \mathrm{~A}$ with site 3 only reconstituted in PDC (Figure 5). PDKs demonstrated different activities towards the three phosphorylation sites. For site 1 PDK2 had the maximum level of activity $(125 \pm 6 \mathrm{mU} / \mathrm{mg})$, followed by PDK4 (70 $\pm 3 \mathrm{mU} / \mathrm{mg})$, PDK1 $(63 \pm 4 \mathrm{mU} / \mathrm{mg})$ and PDK3 $(36 \pm 0.5 \mathrm{mU} / \mathrm{mg})$. For site 2 the activity was: PDK3 > PDK4 > PDK2 > PDK1. PDK3 had higher activity towards site 2 than site 1 in reconstituted PDC. PDK1 was the only isoenzyme having activity with site $3(24 \pm 4 \mathrm{mU} /$ $\mathrm{mg}$ ) that was similar to its activity for site 2 (Korotchkina and Patel, 2001b). The absence of activity of PDK2, PDK3 and PDK4 for site 1 was demonstrated recently in another independent study (Kolobova et al., 2001). PDK3 had the lowest apparent $\mathrm{K}_{\mathrm{m}}$ value for ATP and $S_{0.5}$ values for E1s (with site 1 or site 2 only) and the highest catalytic efficiency $\left(\mathrm{k}_{\mathrm{cat}} / \mathrm{S}_{0.5}\right)$ for both site 1 and site 2 (and also highest catalytic efficiency for ATP) compared with the other three PDK isoenzymes (Korotchkina and Patel, 2001b).

Activities of PDKs depend upon the proportion of oxidized and reduced plus acetylated lipoyl groups of E2 in reconstituted PDC (Ravindran et al., 1996). Figure 5
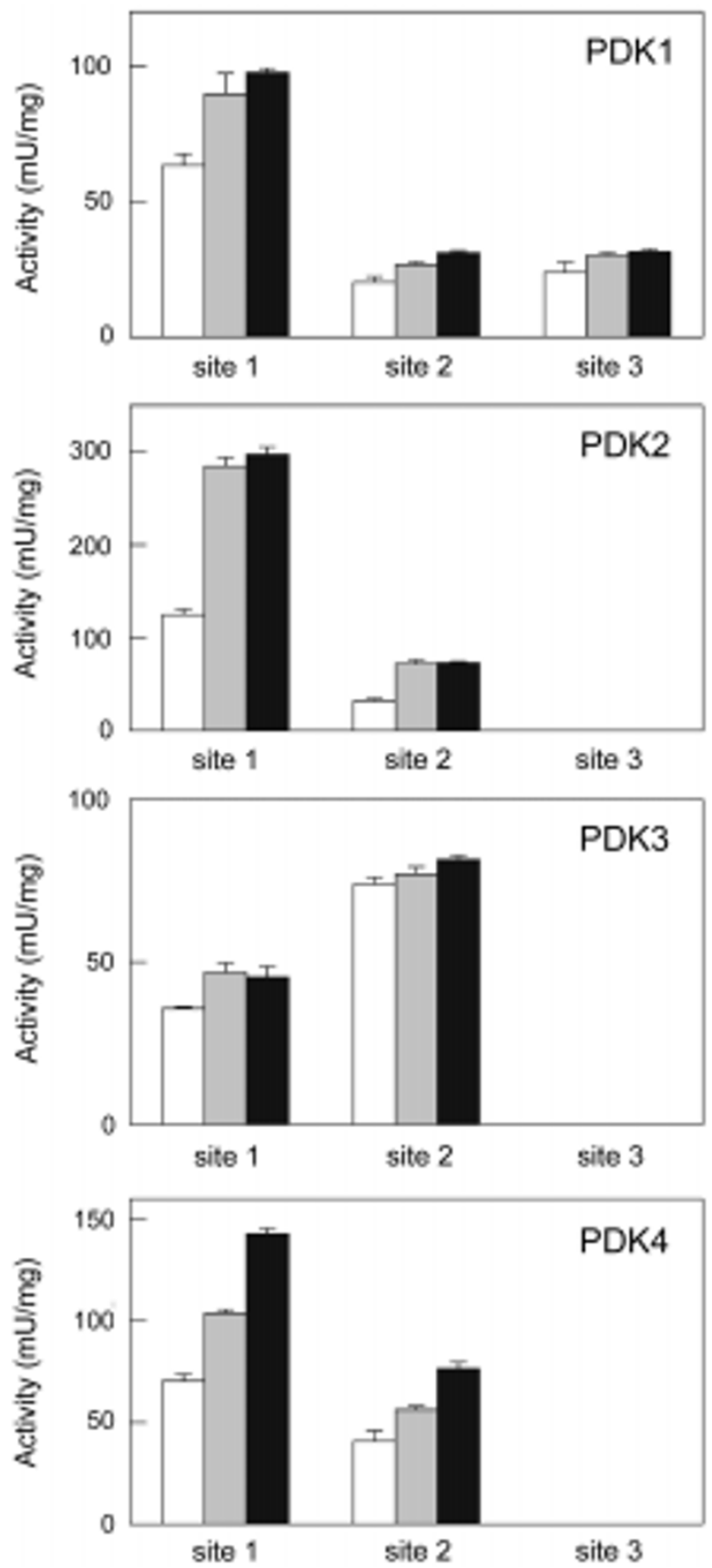

Figure 5. Activities of PDKs towards site-specific mutant E1s reconstituted in PDC in phosphate buffer. Activities of PDKs were determined by the incorporation of ${ }^{32} \mathrm{P}$ into $\mathrm{E} 1$ (2A3A for site 1 only, 1A3A for site 2 only, 1A2A for site 3 only) reconstituted with E2-E3BP and E3 (Korotchkina and Patel, 2001b). PDC was preincubated for 5 min with $\mathrm{NAD}^{+} / \mathrm{NADH}=796 \mu \mathrm{M} / 4 \mu \mathrm{M}$ to oxidize lipoyl groups (white bars), NADH/NAD $=600 \mu \mathrm{M} / 200 \mu \mathrm{M}$ and 50 $\mu \mathrm{M} C o \mathrm{~A}$ to reduce lipoyl groups (grey bars) and $\mathrm{NADH} / \mathrm{NAD}^{+}=600 \mu \mathrm{M} / 200$ $\mu \mathrm{M}$ and $50 \mu \mathrm{M}$ acetyl-CoA to reduce and acetylate lipoyl groups (Korotchkina and Patel, 2001b). *, not detectable.

shows the activities of PDK in three experimental conditions of the lipoyl groups of E2: (i) oxidized; (ii) reduced; and (iii) reduced/acetylated in phosphate buffer. PDK2 is 
maximally stimulated by the reduction/acetylation of the lipoyl groups of E2. PDK3 is less sensitive to the products of PDC reaction. The extent of percent stimulation did not significantly differ on the site being phosphorylated.

Binding of PDKs to the lipoyl domains of E2 was shown to increase their catalytic efficiency (Baker et al., 2000). Examination of activities of four PDK isoenzymes towards free $\mathrm{E} 1$ indicated that not only site 1 can be phosphorylated in the absence of E2, but also site 2 by four PDKs and site 3 by PDK1 (at a slightly higher rate than site 2). Maximal fold activation (7-fold for site 1 and 29-fold for site 2) was demonstrated by PDK3 (Korotchkina and Patel, 2001b). PDK4 displayed much higher activity towards site 2 (4-9-fold) than activities of other three PDK isoenzymes (Korotchkina and Patel, 2001b). These facts could be of the physiological importance taking into consideration that the amounts of PDK4 are shown to increase during starvation, diabetes, hyperthyroidism and high-fat feeding (Sugden et al., 2000; Wu et al., 1998). Also, starvation causes the overexpression of PDK2 as well as PDK4 in liver, kidney and lactating mammary gland (Wu et al., 2000). Presence of the free forms of PDKs may increase hyperphosphorylation of PDC in pathological conditions.

\section{Concluding remarks}

The importance of the PDC for carbohydrate metabolism necessitates the complexity of its regulation by phosphorylation. Each of the three phosphorylation sites in mammalian E1 was found to be important for regulation of E1 and hence PDC. The phosphorylation rates of the three sites are different, however, the dephosphorylation mechanism is random. Variable substitutions of site 1 of human E1 demonstrated that both the negative charge and the size of the introduced group affected the PDC activity. The mechanism of inactivation was found to be different due to phosphorylation of each of the three sites. Phosphorylation of site 1 (and probably site 2) has impact on interaction of E1 with its substrates. Most probably phosphorylation affects both reactions of E1, but more severely the second partial reaction, i.e. interaction with the lipoyl domain of E2. Phosphorylation of site 3 impaired TPP-binding more than phosphorylation of the other two sites. Four isoenzymes of PDK have different specificity for the three phosphorylation sites of E1, are activated to a different extent through binding to E2 and are stimulated differently by the products of the PDC reaction (namely $\mathrm{NADH}$ and acetyl-CoA) via the reduction/acetylation of the lipoyl groups of E2. The complexity of mechanism of PDC regulation by phosphorylationdephosphorylation emphasizes the importance of the tissue-specific modulation of PDC activity in normal and pathological conditions.

\section{Acknowledgments}

This work was supported by U. S. Public Health Service Grant DK20478.

\section{References}

Aevarsson A, Chuang JL, Wynn RM, Turley S, Chuang DT, Hol WGJ. Crystal structure of human branched-chain $\alpha$ ketoacid dehydrogenase and the molecular basis of multienzyme complex deficiency in maple syrup urine disease. Structure 2000;8:277-91

Aevarsson A, Seger K, Turley S, Sokatch JR, Hol WG. Crystal structure of 2-oxoisovalerate and dehydrogenase and the architecture of 2-oxo acid dehydrogenase multienzyme complexes. Nat Struct Biol 1999;6:785-92

Baker JC, Yan X, Peng T, Kasten S, Roche TE. Marked differences between two isoforms of human pyruvate dehydrogenase kinase. J Biol Chem 2000;275:15773-81

Bowker-Kinley MM, Davis WI, Wu P, Harris RA, Popov KM. Evidence for existence of tissue-specific regulation of the mammalian pyruvate dehydrogenase complex. Biochem $\mathrm{J}$ 1998;329:191-96

Bowker-Kinley M, Popov KM. Evidence that pyruvate dehydrogenase kinase belongs to the ATPase/kinase superfamily. Biochem J 1999;344:47-53

Butler JR, Pettit RH, Davis PF, Reed LJ. Binding of thiamin thiazolone pyrophosphate to mammalian pyruvate dehydrogenase and its effects of kinase and phosphatase activities. Biochem Biophys Res Commun 1977;74:1667-74

Chen W, Huang X, Komuniecki PR, Komuniecki R. Molecular cloning, functional expression, and characterization of pyruvate dehydrogenase kinase from anaerobic muscle of the parasitic nematode Ascaris suum. Arch Biochem Biophys 1998;353:181-89

Dahl HH, Hunt SM, Hutchison WM, Brown GK. The human pyruvate dehydrogenase complex. Isolation of cDNA clones for the E1 alpha subunit, sequence analysis, and characterization of the mRNA. J Biol Chem 1987;262:7398-403

Harris RA, Huang B, Wu P. Control of pyruvate dehydrogenase kinase gene expression. Adv Enzyme Regul 2001;41: 269-88

Huang YJ, Walker D, Chen W, Klingbeil M, Komuniecki R. Expression of pyruvate dehydrogenase isoforms during the aerobic/anaerobic transition in the development of the parasitic nematode Ascaris suum: altered stoichiometry of phosphorylation/inactivation. Arch Biochem Biophys 1998;352:26370

Katsube T, Nomoto S, Togashi S, Ueda R, Kobayashi M, Takahisa M. cDNA sequence and expression of a gene encoding a pyruvate dehydrogenase kinase homolog of Drosophila melanogaster. DNA Cell Biol 1997;16:335-39

Khailova LS, Korochkina LG. Determination of the number of active centers in the pyruvate dehydrogenase component of 
the pyruvate dehydrogenase complex from pigeon breast muscle. Biochem Intern 1982;5:525-32

Kolobova E, Tuganova A, Boulatnikov I, Popov KM. Regulation of pyruvate dehydrogenase activity through phophorylation at multiple sites. Biochem J 2001;358:69-77

Korotchkina LG, Khailova LS, Severin SE. The effect of phosphorylation on pyruvate dehydrogenase. FEBS Lett 1995;364: 185-88

Korotchkina LG, Patel MS. Mutagenesis studies of the phosphorylation sites of recombinant human pyruvate dehydrogenase. Site-specific regulation. J Biol Chem 1995;270:14297304

Korotchkina LG, Patel MS. Probing the mechanism of inactivation of human pyruvate dehydrogenase by phosphorylation of three sites. J Biol Chem 2001a;276:5731-38

Korotchkina LG, Patel MS. Site specificity of four pyruvate dehydrogenase kinase isoenzymes toward the three phosphorylation sites of human pyruvate dehydrogenase. J Biol Chem 2001b;276:37223-29

Liu S, Gong X, Yan X, Peng T, Baker JC, Li L, Rodden PM, Ravindran S, Andersson LA, Cole AB, Roche TE. Reaction mechanism for mammalian pyruvate dehydrogenase using natural lipoyl domain substrates. Arch Biochem Biophy 2001; 386:123-35

Nemerya NS, Zemskova MA, Nyukhalkina IA, Khailova LS. Chemical modification of the essential arginine residues of pyruvate dehydrogenase prevents its phosphorylation by kinase. FEBS Lett 1996;394:96-98

Patel MS, Harris RA. Mammalian alpha-keto acid dehydrogenase complexes: gene regulation and genetic defects. FASEB J 1995;9:1164-72

Patel MS, Roche TE. Molecular biology and biochemistry of pyruvate dehydrogenase complexes. FASEB J 1990;4:3224-33

Perham RN. Swinging arms and swinging domains in multifunctional enzymes: catalytic machines for multistep reactions. Annu Rev Biochem 2000;69:961-1004

Ravindran S, Radke GA, Guest JR, Roche TE. Lipoyl domainbased mechanism for the integrated feedback control of the pyruvate dehydrogenase complex by enhancement of pyruvate dehydrogenase kinase activity. J Biol Chem 1996;271: 653-62

Reed L. J. A trail of research from lipoic acid to $\alpha$-keto acid dehydrogenase complexes. J Biol Chem 2001;276:38329-36

Roche TE, Baker JC, Yan X, Hiromasa Y, Gong X, Peng T, Dong J, Turkan A, Kasten SA. Distinct regulatory properties of pyruvate dehydrogenase kinase and phosphatase isoforms. Progr Nucleic Acid Res Molec Biol 2001;70:33-75

Roche TE, Reed LJ. Function of the nonidentical subunits of mammalian pyruvate dehydrogenase. Biochem Biophys Res Commun 1972;48:840-46

Rowles J, Scherer SW, Xi T, Majer M, Nickle DC, Rommens JM., Popov KM, Harris RA, Riebow NL, Xia J, Tsui LC,
Bogardus C, Prochazka M. Cloning and characterization of PDK4 on 7q21.3 encoding a fourth pyruvate dehydrogenase kinase isoenzyme in human. J Biol Chem 1996;271:22376-82

Sale GJ, Randle PJ. Occupancy of phosphorylation sites in pyruvate dehydrogenase phosphate complex in rat heart in vivo. Relation to proportion of inactive complex and rate of reactivation by phosphatase. Biochem J 1982;206:221-29

Sugden MC, Holness MJ. Interactive regulation of the pyruvate dehydrogenase complex and the carnitine palmitoyltransferase system. FASEB J 1994;8:54-61

Sugden MC, Langdown ML, Harris RA, Holness MJ. Expression and regulation of pyruvate dehydrogenase kinase isoforms in the developing rat heart and in adulthood: role of thyroid hormone status and lipid supply. Biochem J 2000;352: 731-38

Sugden PH, Randle PJ. Regulation of pig heart pyruvate dehydrogenase by phosphorylation. Studies on the subunit and phosphorylation stoicheiometries. Biochem J 1978;173: 659-68

Thelen JJ, Miernyk JA, Randall DD. Partial purification and characterization of the maize mitochondrial pyruvate dehydrogenase complex. Plant Physiol 1998;116:1443-50

Thelen JJ, Miernyk JA, Randall DD. Pyruvate dehydrogenase kinase from Arabidopsis thaliana: a protein histidine kinase that phosphorylates serine residues. Biochem J 2000;349: 195-201

Walsh DA, Cooper RH, Denton RM, Bridges BJ, Randle PJ. The elementary reactions of the pig heart pyruvate dehydrogenase complex. A study of the inhibition by phosphorylation. Biochem J 1976;157:41-67

Wu P, Blair PV, Sato J, Jaskiewicz J, Popov KM, Harris RA.Starvation increases the amount of pyruvate dehydrogenase kinase in several mammalian tissues. Arch Biochem Biophys 2000;381:1-7

Wu P, Sato J, Zhao Y, Jaskiewicz J, Popov KM, Harris RA. Starvation and diabetes increase the amount of pyruvate dehydrogenase kinase isoenzyme 4 in rat heart. Biochem $\mathrm{J}$ 1998;329:197-201

Yang D, Gong X, Yakhnin A, Roche TE. Requirements for the adaptor protein role of dihydrolipoyl acetyltransferase in the up-regulated function of the pyruvate dehydrogenase kinase and pyruvate dehydrogenase phosphatase. J Biol Chem 1998;273:14130-37

Yeaman SJ, Hutcheson ET, Roche TE, Pettit FH, Brown JR, Reed LJ, Watson DC, Dixon GH. Sites of phosphorylation on pyruvate dehydrogenase from bovine kidney and heart. Biochemistry 1978;17:2364-70

Zhao Y, Hawes J, Popov KM, Jaskiewicz J, Shimomura Y, Crabb DW, Harris RA. Site-directed mutagenesis of phosphorylation sites of the branched chain alpha-ketoacid dehydrogenase complex. J Biol Chem 1994;269:18583-87 